\title{
Analytical investigation of the orbital structure close to the 1:1:1 resonance in spheroidal galaxies
}

\author{
S. Breiter ${ }^{1}$, A. Elipe ${ }^{2}$, and I. Wytrzyszczak ${ }^{1}$ \\ 1 Astronomical Observatory of A. Mickiewicz University, Słoneczna 36, 60-286 Poznań, Poland \\ e-mail: [breiter;iwona]@amu.edu.pl \\ 2 Grupo de Mecánica Espacial, Universidad de Zaragoza, 50009 Zaragoza, Spain \\ e-mail: elipe@unizar.es
}

Recieved 2 July 2004 / Accepted 18 October 2004

\begin{abstract}
The motion of stars in prolate and oblate spheroidal galaxies is studied. Using a combination of Lissajous and Poincaré transformations, we introduce the set of action-angle-like variables. Then, we perturb the problem of the 1:1:1 resonance motion in a spherical, homogenous galaxy, assuming the perturbing potential in the polynomial Ferrers form. We find the orbits that are periodic with respect to the radial and vertical oscillations by inspecting the equilibria of a normalized system. Imposing additional constraints, we find orbits that are periodic in all three coordinates with respect to the system that rotates with a galaxy.
\end{abstract}

Key words. celestial mechanics - methods: analytical - galaxies: kinematics and dynamics

\section{Introduction}

The question of periodic orbits is often raised in generally nonintegrable dynamical problems. Looking for the types of periodic motion in elliptical galaxies, both numerical and analytical methods are being applied. Classical papers like Contopoulos (1960) or Ollongren (1962), treat the problem by inspecting the motion in the rotating cylindrical coordinates co-moving with a star's orbit guiding centre. This approach, however, becomes problematic if a star approaches the symmetry axis of the galaxy. More general results obtained by Davoust (1983, 1986), using the Cartesian coordinates in a reference frame rotating with an arbitrary rate, support the Lindstedt method as the best-suited tool for the perturbative analytical approach in the hunt for periodic orbits. Averaging techniques, however, have also been used (cf. Gustavson 1966; de Zeeuw 1985). Recently, Jalali \& Sobouti (1998) proposed the application of the canonical transformation method that hinges upon the use of the so-called Lissajous variables invented by Deprit (1991). The difference between the two methods can be best summarized as the the opposition of the epicyclic formulation in the Lindstedt case versus the osculating ellipse approach in the Lissajous variables.

In the present paper we adopt a similar Lissajous-type approach, although a slightly different set of variables and a different parametrization and partition of the Hamiltonian function - both presumably more convenient - has been introduced. The results to be presented are restricted to the spheroidal galaxies described by the Ferrers potential. Our choice of the variables requires that both the radial and longitudinal oscillations are short-period and the difference in their phases evolves slowly. This assumption implies 1:1 resonance and thus restricts the possible types of periodic orbits. We introduce the rotation rate of the reference frame as a free parameter that eventually can be equal to the rotation rate of the galaxy. We study the equilibria of the normalized Hamiltonian system with one degree of freedom and establish their stability and bifurcations. This provides a global look at possible types of motion in the galaxy, but also serves as the key to the search for periodic orbits. The fact that periodic orbits inherit the stability properties of the equilibria that generate them is a significant advantage over the Lindstedt approach.

\section{The galactic potential}

We consider spheroidal galaxies with a density $\rho$ specified by the special case of Ferrers' law of mass distribution (Ferrers 1877; Binney \& Tremaine 1987): inside a galaxy it is given by

$\rho=\rho_{0}\left(1-\frac{x^{2}+y^{2}}{a^{2}}-\frac{z^{2}}{c^{2}}\right)$ 
while outside the galaxy $\rho=0$. Equatorial and polar semimajor axes of the spheroid are $a$ and $c$, respectively. The gravitational potential at an arbitrary interior point is then given by

$V=-\frac{\pi \kappa \rho_{0} a^{2} c}{2} \int_{0}^{\infty} \frac{\left(1-\frac{x^{2}+y^{2}}{u+a^{2}}-\frac{z^{2}}{u+c^{2}}\right)^{2}}{\left(u+a^{2}\right) \sqrt{u+c^{2}}} \mathrm{~d} u$,

where $u \geqslant 0$ marks successive equipotential surfaces.

Substituting $c=a$ and performing integration, we can obtain Ferrers' potential for an inhomogeneous spherical galaxy

$V_{\mathrm{s}}=\pi \kappa \rho_{0} r^{2}\left(\frac{2}{3}-\frac{1}{5} \frac{r^{2}}{a^{2}}\right)$,

depending only on the radial distance from the center of mass $r=\sqrt{x^{2}+y^{2}+z^{2}}$, the reference density $\rho_{0}$, and the gravity constant $\kappa$ (Davoust 1983). We consider $V_{\mathrm{s}}$ as the sum of the main quadratic term due to a homogeneous mass distribution

$V_{0}=\frac{2}{3} \pi \kappa \rho_{0} r^{2}$

and a quartic perturbation. Thus, the unperturbed potential $V_{0}$ defines an isotropic, three-dimensional harmonic oscillator with the fundamental frequency $\omega_{0}$

$\omega_{0}^{2}=\frac{4}{3} \pi \kappa \rho_{0}$.

In an inertial reference frame the unperturbed motion occurs in the 1:1:1 resonance.

The equatorial semi-axis $a$ will be considered the unit length. The unit of time is such that $\omega_{0}=1$ regardless of the mass unit. With this choice of units, the value of the gravity constant is

$\kappa=\frac{3}{4 \pi \rho_{0}}$,

in all the expressions of potential.

A dimensionless shape parameter

$e=1-\left(\frac{c}{a}\right)^{2}$,

allows substitution $c=a \sqrt{1-e}$ and is valid for both oblate $(0<e<1)$ and prolate $(-\infty<e<0)$ galaxies. It is different from the commonly used shape parameters, but - in spite of the apparent asymmetry - its application renders relatively simple equations that share the same form regardless of the oblate or the prolate case.

Using the new units, one can write the Ferrers' potential (2) as

$$
\begin{aligned}
V & =-\frac{3 \sqrt{1-e}}{8} \int_{0}^{\infty} \frac{\left(1-\frac{x^{2}+y^{2}}{u+1}-\frac{z^{2}}{u+1-e}\right)^{2}}{(u+1) \sqrt{u+1-e}} \mathrm{~d} u \\
& =\frac{r^{2}}{2}-\frac{3}{20} r^{4}+O(e) .
\end{aligned}
$$

Performing all products we find the explicit, polynomial form of $V$

$$
\begin{aligned}
V= & V_{0}-\left(A_{1}\left(x^{2}+y^{2}-2 z^{2}\right)\right. \\
& \left.+C_{1}\left(x^{2}+y^{2}\right) z^{2}+C_{2}\left(x^{2}+y^{2}\right)^{2}+C_{3} z^{4}\right)
\end{aligned}
$$

Table 1. Potential coefficients.

\begin{tabular}{cccc}
\hline \hline Coefficient & $e \rightarrow-\infty$ & $e \approx 0$ & $e \rightarrow 1$ \\
\hline$A_{1}$ & $-\frac{1}{4}$ & $\frac{1}{10} e+O\left(e^{2}\right)$ & $\frac{1}{2}$ \\
$C_{1}$ & 0 & $\frac{3}{10}+\frac{6}{35} e+O\left(e^{2}\right)$ & $\frac{3}{2}$ \\
$C_{2}$ & $\frac{3}{16}$ & $\frac{3}{20}-\frac{3}{140} e+O\left(e^{2}\right)$ & 0 \\
$C_{3}$ & 0 & $\frac{3}{20}+\frac{27}{140} e+O\left(e^{2}\right)$ & $-\infty$ \\
\hline
\end{tabular}

where

$A_{1}=\frac{1}{2}-\int_{0}^{\infty} \frac{3(1-e)^{\frac{1}{2}} \mathrm{~d} u}{4(1+u)^{2}(1-e+u)^{\frac{1}{2}}}$,
$C_{1}=\int_{0}^{\infty} \frac{3(1-e)^{\frac{1}{2}} \mathrm{~d} u}{4(1+u)^{2}(1-e+u)^{\frac{3}{2}}}$,

$C_{2}=\int_{0}^{\infty} \frac{3(1-e)^{\frac{1}{2}} \mathrm{~d} u}{8(1+u)^{3}(1-e+u)^{\frac{1}{2}}}$,

$C_{3}=\int_{0}^{\infty} \frac{3(1-e)^{\frac{1}{2}} \mathrm{~d} u}{8(1+u)(1-e+u)^{\frac{5}{2}}}$.

All integrals in (10) are elementary. Moreover, an integration by parts provides simple relations between the shape dependent coefficients

$A_{1}=\frac{1}{3} e C_{1}$,

$C_{2}=\frac{3}{16}-\frac{1}{8} C_{1}$,

$C_{3}=\frac{1}{4}(1-e)^{-1}-\frac{1}{3} C_{1}$,

where $C_{1}$ can be expressed in terms of elementary functions

$C_{1}=\frac{9}{4 e^{2}}\left(1-\frac{e}{3}-\sqrt{1-e} \alpha(e)\right)$,

with

$\alpha(e)= \begin{cases}e^{-\frac{1}{2}} \arcsin \sqrt{e} & \text { for } e \geqslant 0, \\ |e|^{-\frac{1}{2}} \operatorname{arsinh} \sqrt{|e|} & \text { for } e \leqslant 0,\end{cases}$

or, more conveniently, as a Gaussian hypergeometric function

$C_{1}=\frac{3}{10}{ }_{2} F_{1}(2,4 ; 7 / 2 ;(1-\sqrt{1-e}) / 2)$.

Table 1 provides asymptotic and approximate values of the coefficients.

The perturbing potential is invariant with respect to the rotations around the $z$ axis. Its magnitude depends on two factors: the galactic oblateness parameter $e$, and the ratio $(r / a)^{4}$.

\section{Hamiltonian and canonical transformations}

In order to study the motion of an object inside a spheroidal galaxy, we introduce a reference frame rotating with the angular rate $\Omega$ around the symmetry axis of the galaxy.

The Hamiltonian $\mathcal{H}$ is composed of two parts: the unperturbed $\mathcal{H}_{0}$ describes the motion in a reference galaxy that is 
homogeneous and spherical, considered in the rotating frame, and $\mathcal{H}_{1}$ is a perturbing potential

$$
\begin{aligned}
\mathcal{H}= & \mathcal{H}_{0}+\mathcal{H}_{1}, \\
\mathcal{H}_{0}= & \frac{1}{2}\left(X^{2}+Y^{2}+Z^{2}+x^{2}+y^{2}+z^{2}\right)-\Omega(x Y-y X), \\
\mathcal{H}_{1}= & -A_{1}\left(x^{2}+y^{2}-2 z^{2}\right)-C_{1}\left(x^{2}+y^{2}\right) z^{2} \\
& -C_{2}\left(x^{2}+y^{2}\right)^{2}-C_{3} z^{4} .
\end{aligned}
$$

The Hamiltonian $\mathcal{H}_{0}$ allows a complete reduction to actionangle variables. We achieve it by means of three subsequent canonical transformations. The first step is the Lissajous transformation (Deprit 1991) that takes the advantage of the fact that the system consisting of two unperturbed oscillators in the $x, y$ plane and rotating around the $z$ axis possesses the visviva integral

$L=\frac{1}{2}\left(X^{2}+Y^{2}\right)+\frac{1}{2}\left(x^{2}+y^{2}\right)$,

and the integral of the $z$ component of orbital angular momentum

$G=x Y-y X$.

The third integral is related to the vertical oscillations

$H=\frac{1}{2}\left(Z^{2}+z^{2}\right)$,

hence, the second step is a classical Poincare transformation for the $(z, Z)$ pair with the action variable $H$. The two transformations are defined as

$X=-s \sin (l+g)-d \sin (g-l)$,

$Y=s \cos (l+g)+d \cos (g-l)$,

$Z=\sqrt{2 H} \cos h$,

$x=s \cos (l+g)-d \cos (g-l)$,

$y=s \sin (l+g)-d \sin (g-l)$,

$z=\sqrt{2 H} \sin h$

where

$s=\sqrt{\frac{L+G}{2}}, \quad d=\sqrt{\frac{L-G}{2}}$.

The unperturbed Hamiltonian $\mathcal{H}_{0}$ expressed in terms of the new Lissajous-Poincaré variables becomes

$\mathcal{H}_{0}=L+H-\Omega G$.

Noticing the degeneracy of $\mathcal{H}_{0}$ with respect to the frequencies $\dot{l}=\dot{h}=1$, we introduce a third transformation

$\Phi=L, \quad \Psi=L+H, \quad \phi=l-h, \quad \psi=h$.
Finally, the Cartesian coordinates and momenta become

$$
\begin{aligned}
& X=-s \sin (\phi+\psi+g)+d \sin (\phi+\psi-g), \\
& Y=s \cos (\phi+\psi+g)+d \cos (\phi+\psi-g), \\
& Z=\sqrt{2(\Psi-\Phi)} \cos \psi, \\
& x=s \cos (\phi+\psi+g)-d \cos (\phi+\psi-g), \\
& y=s \sin (\phi+\psi+g)+d \sin (\phi+\psi-g), \\
& z=\sqrt{2(\Psi-\Phi)} \sin \psi,
\end{aligned}
$$

where

$s=\sqrt{\frac{\Phi+G}{2}}, \quad d=\sqrt{\frac{\Phi-G}{2}}$.

The Hamiltonian expressed in terms of the new variables $\psi, \phi$, $g, \Psi, \Phi, G$, is the sum of

$$
\begin{aligned}
\mathcal{H}_{0}= & \Psi-\Omega G \\
\mathcal{H}_{1}= & M_{0}+M_{1} \cos 2 \phi+M_{2} \cos 2 \psi+M_{3} \cos 4 \psi \\
& +M_{4} \cos (2 \psi+2 \phi)+M_{5} \cos (4 \psi+2 \phi) \\
& +M_{6} \cos (4 \psi+4 \phi),
\end{aligned}
$$

where $M_{j}$ are functions of momenta $\Psi, \Phi, G$. Thanks to the axial symmetry, the angle $g$ is cyclic and thus $G$ is a constant of motion.

\section{Geometrical interpretation of variables}

Before we proceed to the normalization of $\mathcal{H}$, let us briefly discuss the geometrical meaning of the variables introduced in the previous section. The orbital motion around the center of a galaxy is decomposed into the equatorial projection (initially described in terms of $l, g, L, G$ ) and the vertical component.

The vertical component amounts to simple harmonic oscillations having the amplitude $\sqrt{2 H}$ (hence $H \geqslant 0$ ) and the phase $h$ is measured from the passage through the equatorial plane $z=0$ with $Z>0$.

The equatorial projection of an unperturbed orbit in the fixed frame is an ellipse with the eccentricity depending on the ratio $|G| / L$. The angle $g$ determines the short $(G>0)$ or the long $(G<0)$ axis orientation of the ellipse, whereas $l$, measured from this axis, serves as a parameter in the parametric equation of the ellipse - quite similarly to the argument of pericenter and eccentric anomaly in the Keplerian problem. The value of $G$ establishes the lower limit of $L$, due to the condition $|G| \leqslant L$. If $L=|G|$, the shape of the equatorial projection is circular and only the sum or the difference of $l$ and $g$ are properly defined. Note that in the rotating reference frame, the ellipse rotates with the angular rate $\dot{g}=-\Omega$. The meaning of $G=0$ can be twofold: either the absence of the total angular momentum (vertical orbits passing through the center of galaxy, recognized by $L=0$ ) or the collapse of the equatorial projection to a straight segment (an elliptic orbit perpendicular to the galactic equator with $L>0$ ).

The final variables reflect the properties of the LissajousPoincaré set. The value of $\Phi$ is bounded by

$|G| \leqslant \Phi \leqslant \Psi$. 
The special cases are: $|G|=\Phi$ for a circular equatorial projection, $\Phi=\Psi$ for the motion taking place in the $z=0$ plane (due to $H=0$ ), $\Phi=0$ for the straight, vertical oscillations (note that $\Phi=0$ necessarily implies $G=0), G=0 \neq \Phi$ for elliptic polar orbits, and finally $G=\Phi=\Psi=0$ for an object at rest in the center of the galaxy. The variable $\phi$ is the resonant angle of our problem. If its value is constant, the equatorial projection of the distance and the $z$ coordinate are synchronized.

To allow a comparison with the traditional epicyclic formulation of motion, we point out few basic facts: in the planar, equatorial case, the guiding center rotates with an angular rate equal to the average of $\dot{l}+\dot{g}$, i.e. of $\dot{\psi}+\dot{\phi}+\dot{g}$; the equatorial epicyclic frequency is given as the average of $2 \dot{l}$, i.e. of $2(\dot{\psi}+\dot{\phi})$. The vertical epicyclic oscillations are directly described in terms of the phase $\psi$. The distance $r$ is given by

$r^{2}=\Psi-(\Psi-\Phi) \cos 2 \psi-\sqrt{\Phi^{2}-G^{2}} \cos 2(\psi+\phi)$,

and its equatorial projection can be obtained if we set $\Psi=\Phi$.

The angular momentum vector

$\boldsymbol{K}=\boldsymbol{r} \times \boldsymbol{R}=\left(K_{1}, K_{2}, K_{3}\right)^{\mathrm{T}}$,

expressed in the new variables, has components

$$
\begin{aligned}
K_{1}= & \sqrt{\Psi-\Phi}(\sqrt{\Phi-G} \sin (\phi-g) \\
& +\sqrt{\Phi+G} \sin (\phi+g)), \\
K_{2}= & \sqrt{\Psi-\Phi}(\sqrt{\Phi-G} \cos (\phi-g) \\
& -\sqrt{\Phi+G} \cos (\phi+g)), \\
K_{3}= & G .
\end{aligned}
$$

The total momentum $K$ is given by

$K^{2}=G^{2}+2(\Psi-\Phi)\left(\Phi-\sqrt{\Phi^{2}-G^{2}} \cos 2 \phi\right)$.

\section{Normalization}

The essential gain of our three-step transformation is that now the Lie derivative operator associated with $\mathcal{H}_{0}$ is simply

$\mathcal{L}_{0}=\frac{\partial}{\partial \psi}-\Omega \frac{\partial}{\partial g}$

Recalling that $g$ is cyclic, we can drop the second term of Eq. (35) in most of the applications. In these circumstances no difficulties are met when we attempt the normalization of the system. Following the method of Deprit (1969), we define a canonical transformation such that the Hamiltonian $\mathcal{H}$, expressed in terms of some new "mean" variables, becomes a function $\mathcal{K}$ belonging to the kernel of $\mathcal{L}_{0}$. If we stop at the first order of perturbations, the transformation

$\Xi=\{\psi, \phi, g, \Psi, \Phi, G\} \quad \leftrightarrows \quad \Xi^{*}=\left\{\psi^{*}, \phi^{*}, g^{*}, \Psi^{*}, \Phi^{*}, G^{*}\right\}$

is defined in terms of the Lie generator $\mathcal{W}$

$$
\begin{aligned}
\mathcal{W}(\Xi)= & \frac{M_{2}}{2} \sin 2 \psi+\frac{M_{3}}{4} \sin 4 \psi+\frac{M_{4}}{2} \sin (2 \psi+2 \phi) \\
& +\frac{M_{5}}{4} \sin (4 \psi+2 \phi)+\frac{M_{6}}{4} \sin (4 \psi+4 \phi) .
\end{aligned}
$$

Note that $\mathcal{W}$ does not depend on $g$ and so

$G=G^{*}+\left(G^{*} ; \mathcal{W}\left(\Xi^{*}\right)\right)=G^{*}$,

where ( ; ) stands for the Poisson bracket.

The transformed Hamiltonian $\mathcal{K}\left(\Xi^{*}\right)=\mathcal{K}_{0}+\mathcal{K}_{1}$ is the sum of

$$
\begin{aligned}
\mathcal{K}_{0}= & \Psi^{*}-\Omega G \\
\mathcal{K}_{1}= & M_{0}+M_{1} \cos 2 \phi^{*} \\
= & A_{1}\left(2 \Psi^{*}-3 \Phi^{*}\right)-C_{1} \Phi^{*}\left(\Psi^{*}-\Phi^{*}\right) \\
& -\frac{1}{2} C_{2}\left(3\left(\Phi^{*}\right)^{2}-G^{2}\right)-\frac{3}{2} C_{3}\left(\Psi^{*}-\Phi^{*}\right)^{2} \\
& -\frac{1}{2} C_{1}\left(\Psi^{*}-\Phi^{*}\right) \sqrt{\left(\Phi^{*}\right)^{2}-G^{2}} \cos 2 \phi^{*} .
\end{aligned}
$$

The reduced system defined by $\mathcal{K}$ has only one degree of freedom and the motion on a "mean orbit" is now governed by canonical equations

$$
\begin{aligned}
\dot{\psi}^{*}= & 1+2 A_{1}-3 C_{3}\left(\Psi^{*}-\Phi^{*}\right)-C_{1} \Phi^{*} \\
& -\frac{C_{1}}{2} \sqrt{\left(\Phi^{*}\right)^{2}-G^{2}} \cos 2 \phi^{*}, \\
\dot{\Psi}^{*}= & 0, \\
\dot{g}^{*}= & -\Omega+C_{2} G+C_{1} G \frac{\Psi^{*}-\Phi^{*}}{\sqrt{\left(\Phi^{*}\right)^{2}-G^{2}}} \cos 2 \phi^{*}, \\
\dot{G}= & 0, \\
\dot{\phi}^{*}= & -3 A_{1}-C_{1}\left(\Psi^{*}-2 \Phi^{*}\right)+3 C_{3}\left(\Psi^{*}-\Phi^{*}\right) \\
& -3 C_{2} \Phi^{*}+\frac{C_{1}}{2} \frac{2\left(\Phi^{*}\right)^{2}-\Psi^{*} \Phi^{*}-G^{2}}{\sqrt{\left(\Phi^{*}\right)^{2}-G^{2}}} \cos 2 \phi^{*}, \\
\dot{\Phi}^{*}= & -C_{1}\left(\Psi^{*}-\Phi^{*}\right) \sqrt{\left(\Phi^{*}\right)^{2}-G^{2}} \sin 2 \phi^{*} .
\end{aligned}
$$

It is worth noting that a new integral of motion has been created. It reads $\mathcal{K}_{0}\left(\Psi^{*}, G\right)=$ const., or simply $\Psi^{*}=$ const. In practice, the integral is approximate, because we know the transformation from $\Psi^{*}$ to the original $\Psi$ only up to the first order

$\Psi^{*} \approx \Psi-\left\{\Psi, W_{1}(\Xi)\right\} \approx \mathcal{H}(\Xi)-\mathcal{K}_{1}(\Xi)+\Omega G$.

Observe that $\mathcal{H}(\Xi)$ is exactly conserved, which brings us to the statement that $\mathcal{K}_{1}(\Xi) \approx$ const. is an approximate integral. Is it the usual "third integral" (Contopoulos 1960) of the stellar dynamics? The answer is: not yet, but indeed we recognize in $\mathcal{K}_{1}$ the first guess for the Whittaker (1944) procedure applied by Contopoulos \& Moutsoulas (1965), or for the Birkhoff procedure applied by Gustavson (1966). The problem is that $\mathcal{K}_{1}$ is already a first order quantity with a second order remainder that is not constant; thus, effectively, $\mathcal{K}_{1}(\Xi) \approx$ const. is a statement accurate only at the zero order of the perturbation technique, i.e. merely the first guess.

\section{Equilibria in $\left(\phi^{*}, \Phi^{*}\right)$ and in other related variables}

Throughout this section only the mean orbits are discussed and for the sake of brevity we will suppress the asterisks over all variables. Our first goal is to find all equilibria of the coupled Eqs. (44) and (45). 
The right hand side of Eq. (45) has $\sin 2 \phi$ as a factor that vanishes if $\phi_{\mathrm{c}}=k \frac{\pi}{2}$, with $k \in\{0,1,2,3\}$. In that case, $\cos 2 \phi$ in Eq. (44) takes the value of 1 , if $k \in\{0,2\}$, or -1 if $k \in\{1,3\}$, leading to the equilibrium conditions for $\Phi$

$$
\begin{aligned}
-3 A_{1}-\left(C_{1}-3 C_{3}\right) \Psi+ & \left(2 C_{1}-3 C_{2}-3 C_{3}\right) \Phi \\
\pm & \frac{C_{1}}{2} \frac{2 \Phi^{2}-\Psi \Phi-G^{2}}{\sqrt{\Phi^{2}-G^{2}}}=0 .
\end{aligned}
$$

The possibility of $\dot{\Phi}=0$ due to $\Phi=\Psi$ or $\Phi=|G|$ should not be investigated in terms of the polar variables $(\phi, \Phi)$, because the Hamiltonian is not analytic there and another set of variables will be required for a correct study.

Unfortunately, conditions (47) are equivalent to quartic equations and obtaining their exact solutions is possible, but quite tedious. In these circumstances, we combine simple considerations of the signs of some quantities with numerical methods of root finding in the domain

$|G|<\Phi<\Psi$.

We begin with the second derivatives of the Hamiltonian $\mathcal{K}_{1}$ at $\phi_{\mathrm{c}}=k \frac{\pi}{2}$. They are

$$
\begin{aligned}
{\left[\frac{\partial^{2} \mathcal{K}_{1}}{\partial \phi^{2}}\right]_{\phi_{\mathrm{c}}}=} & \pm 2 C_{1}(\Psi-\Phi) \sqrt{\Phi^{2}-G^{2}}, \\
{\left[\frac{\partial^{2} \mathcal{K}_{1}}{\partial \Phi^{2}}\right]_{\phi_{\mathrm{c}}}=} & 2 C_{1}-3\left(C_{2}+C_{3}\right) \\
& \pm \frac{C_{1}}{2} \frac{2 \Phi^{3}-3 \Phi G^{2}+\Psi G^{2}}{\left(\Phi^{2}-G^{2}\right)^{\frac{3}{2}}}, \\
{\left[\frac{\partial^{2} \mathcal{K}_{1}}{\partial \phi \partial \Phi}\right]_{\phi_{\mathrm{c}}}=} & 0,
\end{aligned}
$$

where, as usual, the upper sign refers to $k \in\{0,2\}$. One can observe that inside the domain (48) the last term of Eq. (50) is strictly positive, whereas the sum of the remaining terms is strictly negative

$$
\begin{aligned}
& 2 C_{1}-3\left(C_{2}+C_{3}\right)<0, \\
& \frac{C_{1}}{2} \frac{2 \Phi^{3}-3 \Phi G^{2}+\Psi G^{2}}{\left(\Phi^{2}-G^{2}\right)^{\frac{3}{2}}}>0 .
\end{aligned}
$$

The latter inequality can be easily verified after a substitution $\Phi=|G|+\Delta_{1}, \Psi=\Phi+\Delta_{2}$ where both $\Delta_{1}>0$ and $\Delta_{2}>0$ due to (48). Observing that $C_{1}>0$ and $\Phi^{2}-G^{2}>0$, we find

$$
\begin{aligned}
& 2 \Phi^{3}-3 \Phi G^{2}+\Psi G^{2}= \\
& 2 \Delta_{1}^{3}+6 \Delta_{1}^{2}|G|+\left(4 \Delta_{1}+\Delta_{2}\right) G^{2}>0 .
\end{aligned}
$$

The expression

$$
\frac{2 \Phi^{3}-3 \Phi G^{2}+\Psi G^{2}}{\left(\Phi^{2}-G^{2}\right)^{\frac{3}{2}}}
$$

is a monotonous, decreasing function of $\Phi$ in the domain (48). This means that if the root $\Phi$ of Eq. (50) exists, it is a unique, simple root.

These properties are very helpful and have significant consequences for the number and stability of critical points.

\subsection{Equilibrium $E_{1}$}

Let us consider $\phi_{\mathrm{c}} \in\{\pi / 2,3 \pi / 2\}$. According to Eqs. (50) and (53), Eq. (47) has either one or zero roots in the domain (48), because in this case the derivative of (47) is strictly negative. If the root $\Phi_{1}$ exists, we obtain two similar equilibria $E_{1}$ with the same values of $\Phi=\Phi_{1}$ and $\phi_{\mathrm{c}}=\frac{1}{2} \pi$ or $\phi_{\mathrm{c}}=\frac{3}{2} \pi$. For the sake of brevity, we will simply call both points "the $E_{1}$ equilibrium", because they share the same properties.

If $E_{1}$ exists, it is a stable critical point. This conclusion results from an inspection of the second derivatives (50) that form the matrix of variational equations

$$
\left[\begin{array}{c}
\delta \dot{\phi} \\
\delta \dot{\Phi}
\end{array}\right]=\left[\begin{array}{cc}
0 & {\left[\frac{\partial^{2} \mathcal{K}_{1}}{\partial \Phi^{2}}\right]_{E_{1}}} \\
-\left[\frac{\partial^{2} \mathcal{K}_{1}}{\partial \phi^{2}}\right]_{E_{1}} & 0
\end{array}\right]\left[\begin{array}{c}
\delta \phi \\
\delta \Phi
\end{array}\right] .
$$

Both eigenvalues of the Jacobian matrix are purely imaginary, because

$\left[\frac{\partial^{2} \mathcal{K}_{1}}{\partial \Phi^{2}}\right]_{E_{1}}<0$, and $\left[\frac{\partial^{2} \mathcal{K}_{1}}{\partial \phi^{2}}\right]_{E_{1}}<0$.

The condition for the existence of $E_{1}$ is directly related to the bifurcations at the $\Phi=\Psi$ limit, hence it will be considered in the next sections.

The orbits of the $E_{1}$ family have a constant mean inclination to the galactic equator, because when $\phi, \Phi$, and $\Psi$ are constant the total angular momentum (34) and its $z$-component (33) are also constant. Of course, the constant value of the mean inclination can be modulated by short-periodic terms when we come back to the original variables. The orbits cross the equator at the points of their maximum distance from the origin and their orbital plane rotates around the galactic polar axis, so the orbits belong to the $z$-tube class with a barrel-shaped envelope (see Fig. 1).

\subsection{Equilibria $E_{2}$ and $E_{3}$}

Let us now pass to the case of $\phi_{c} \in\{0, \pi\}$. According to the behavior of the second derivative (50), Eq. (47) may have up to two distinct roots. If the two roots $\Phi_{2}<\Phi_{3}$ exist, they correspond to two equilibria $E_{2}$ and $E_{3}$ respectively. As in the previous section, we use the term "equilibrium $E_{2}$ " for a pair of points $\left(\phi_{\mathrm{c}}=0, \Phi=\Phi_{2}\right)$ and $\left(\phi_{\mathrm{c}}=\pi, \Phi=\Phi_{2}\right)$, with a similar convention for $E_{3}$.

The family of orbits at the $E_{2}$ and $E_{3}$ equilibria consists of the trajectories having a constant mean inclination, but - in contrast to the $E_{1}$ family - the minimum of $r$ is reached at the equator. Due to the rotation of the orbital plane, these $z$-tube orbits have an hourglass shaped envelope (see Fig. 1).

The stability of $E_{2}$ and $E_{3}$ is complementary: due to the properties of the second derivative $\frac{\partial^{2} \mathcal{K}_{1}}{\partial \Phi^{2}}$ that increases with $\Phi$, we have either

$\left[\frac{\partial^{2} \mathcal{K}_{1}}{\partial \Phi^{2}}\right]_{E_{2}}>0$, and $\left[\frac{\partial^{2} \mathcal{K}_{1}}{\partial \phi^{2}}\right]_{E_{2}}>0$

or

$\left[\frac{\partial^{2} \mathcal{K}_{1}}{\partial \Phi^{2}}\right]_{E_{3}}<0$, and $\left[\frac{\partial^{2} \mathcal{K}_{1}}{\partial \phi^{2}}\right]_{E_{3}}>0$ 

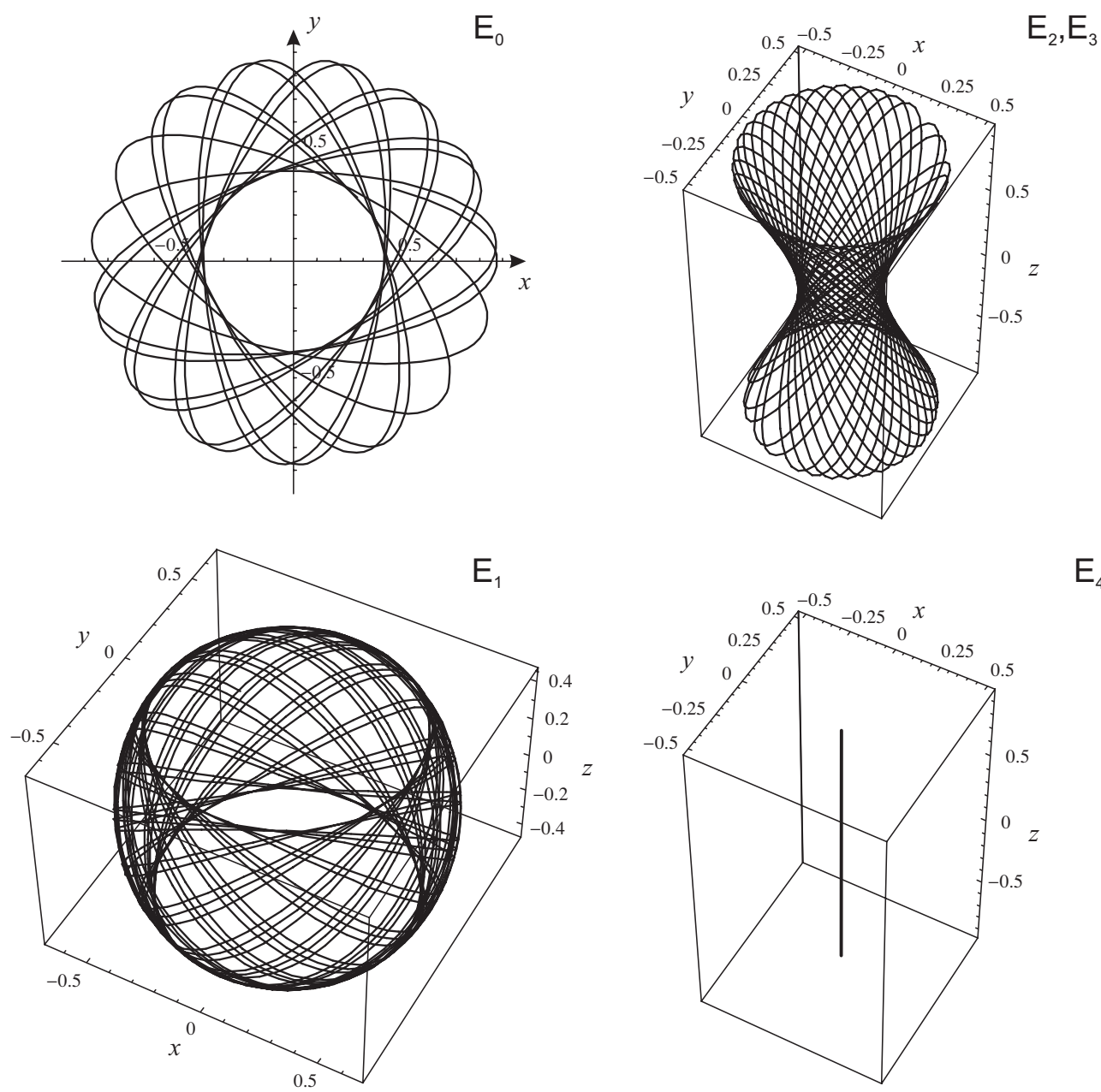

$\mathrm{E}_{4}$

Fig. 1. Examples of orbits related with equilibria $E_{k}$ of the normalized system.

in the Jacobian matrix of variational equations analogous to Eq. (55). As it follows, $E_{2}$ is a stable equilibrium and $E_{3}$ is unstable. Both points may merge at a tangent (saddle-center) bifurcation that occurs for

$2 C_{1}-3\left(C_{2}+C_{3}\right)+\frac{C_{1}}{2} \frac{2 \Phi^{3}-3 \Phi G^{2}+\Psi G^{2}}{\left(\Phi^{2}-G^{2}\right)^{\frac{3}{2}}}=0$.

In that case, the critical point $E_{23}$, a double root of Eq. (47), is an unstable cusp of a homoclinic curve on the $(\phi, \Phi)$ phase plane.

The bifurcation curve on the $G, \Psi$ parametric plane can be obtained by solving a system of two simultaneous Eqs. (47) and (56), the former with a plus sign. The curve is symmetric with respect to $G$. It originates as a cusp at

$G=0, \quad \Psi_{23}=\frac{2 A_{1}}{2 C_{3}-C_{1}}$,

and continues until $\Phi_{2}=\Phi_{3}=\Psi$ - the point of tangency with the bifurcation curve of $E_{0}$ described in the following section.

\subsection{Equilibrium $E_{0}$ - equatorial orbits}

Asking about orbits in the galactic equatorial plane, we need the variables (preferably canonical) that provide an analytic form of $\mathcal{K}_{1}$ when $\Phi=\Psi$. For this purpose we introduce a canonical coordinate-momentum pair $q, Q$ for the one degree of freedom system where $\Psi$ and $G$ are considered as parameters. Under the Poincaré transformation

$q=\sqrt{2(\Psi-\Phi)} \cos \phi, \quad Q=\sqrt{2(\Psi-\Phi)} \sin \phi$,

we obtain the Hamiltonian

$$
\begin{aligned}
\mathcal{K}_{1}= & \frac{q^{2}+Q^{2}}{2}\left[3 A_{1}-\left(C_{1}-3 C_{2}\right) \Psi\right] \\
& +\frac{\left(q^{2}+Q^{2}\right)^{2}}{8}\left[2 C_{1}-3 C_{2}-3 C_{3}\right] \\
& +\frac{C_{1}}{8}\left(Q^{2}-q^{2}\right) \sqrt{\left(2 \Psi-q^{2}-Q^{2}\right)^{2}-4 G^{2}}
\end{aligned}
$$

The Hamiltonian is analytic for equatorial orbits, i.e. at $q=$ $Q=0$. From the equations of motion

$\dot{q}=\frac{\partial \mathcal{K}_{1}}{\partial Q}, \quad \dot{Q}=-\frac{\partial \mathcal{K}_{1}}{\partial q}$,

we find that $q=Q=0$ is an equilibrium. Thus, equatorial orbits with $\Phi=\Psi$ constitute the equilibrium $E_{0}$. In the general case, the $E_{0}$ orbits are rosettes in the equatorial plane, known also as loop orbits, like the one presented in Fig. 1. 
The matrix of variational equations

$$
\left[\begin{array}{c}
\delta \dot{q} \\
\delta \dot{Q}
\end{array}\right]=\left[\begin{array}{cc}
0 & {\left[\frac{\partial^{2} \mathcal{K}_{1}}{\partial Q^{2}}\right]_{E_{0}}} \\
-\left[\frac{\partial^{2} \mathcal{K}_{1}}{\partial q^{2}}\right]_{E_{0}} & 0
\end{array}\right]\left[\begin{array}{c}
\delta q \\
\delta Q
\end{array}\right]
$$

has two eigenvalues $\lambda_{1,2}$ that satisfy

$$
\begin{aligned}
\lambda_{1,2}^{2}= & -\left[\frac{\partial^{2} \mathcal{K}_{1}}{\partial Q^{2}} \frac{\partial^{2} \mathcal{K}_{1}}{\partial q^{2}}\right]_{E_{0}}=-9 A_{1}^{2}+6 A_{1}\left(C_{1}-3 C_{2}\right) \Psi \\
& -\frac{3}{4}\left(C_{1}-6 C_{2}\right)\left(C_{1}-2 C_{2}\right) \Psi^{2}-\frac{C_{1}^{2}}{4} G^{2} .
\end{aligned}
$$

Depending on the sign of the right-hand side of Eq. (62), equatorial orbits are either stable $\left(\lambda_{1,2}^{2}<0\right)$ or unstable $\left(\lambda_{1,2}^{2}>0\right)$. A pitchfork bifurcation occurs when $\lambda_{1,2}^{2}=0$; equating (62) to zero, we define a quadric curve on the parametric plane $(G, \Psi)$ that separates the regions of stable and unstable equatorial orbits. The quadric is symmetric with respect to the $G=0$ line and it intersects the $\Psi$ axis at

$\Psi_{01}=\frac{6 A_{1}}{C_{1}-6 C_{2}}, \quad \Psi_{02}=\frac{2 A_{1}}{C_{1}-2 C_{2}}$.

It is tangent to the $\Psi=|G|$ boundary at $\Psi_{3}=3 A_{1} /\left(C_{1}-3 C_{2}\right)$. For oblate galaxies with $e<0.8$, only $\Psi_{02}$ is positive and the $\Psi=|G|$ points are beyond the limits imposed by the condition that an orbit should not significantly extend outside the galaxy.

As we have signaled in a previous section, the pitchfork bifurcation at $\Phi=\Psi$ is related to the occurrence and disappearance of an equilibrium $E_{1}$ or $E_{3}$.

\subsection{Circular orbits}

"Circular orbits" means the ones with a circular projection onto the galactic equator $(\Phi=|G|)$ - not necessarily the ones with a constant $r$. Similarly to the case of equatorial orbits, we can introduce the Poincaré transformation

$p=\sqrt{\Phi-|G|} \cos 2 \phi, \quad P=-\sqrt{\Phi-|G|} \sin 2 \phi$,

with $p=P=0$ in the $\Phi=|G|$ case. This time, however, the Hamiltonian

$$
\begin{aligned}
\mathcal{K}_{1}= & -\frac{p^{2}+P^{2}}{2}\left[6 A_{1}+2\left(C_{1}-3 C_{3}\right) \Psi\right. \\
& \left.-\left(2 C_{1}-3 C_{2}-3 C_{3}\right)\left(p^{2}+P^{2}+2|G|\right)\right] \\
& +\frac{C_{1}}{2} p\left(p^{2}+P^{2}+|G|-\Psi\right) \sqrt{2|G|+p^{2}+P^{2}},
\end{aligned}
$$

is analytic at $p=P=0$ only for $G \neq 0$. Nevertheless, if $G \neq 0$, circular orbits are not critical points of

$\dot{p}=\left[\frac{\partial \mathcal{K}_{1}}{\partial P}\right]_{0}=0, \quad \dot{P}=-\left[\frac{\partial \mathcal{K}_{1}}{\partial p}\right]_{0}=C_{1}(\Psi-|G|) \sqrt{\frac{|G|}{2}}$

except for the circular-equatorial orbits with $\Psi=\Phi=|G|$. The latter are stable, when the values of $\Psi$ and $G$ imply a stable circular orbit according to the conditions of the stability of $E_{0}$.

\subsection{Equilibrium $E_{4}-$ rectilinear polar orbits}

The special case of $\Phi=G=0$ - rectilinear oscillations along the $z$ axis - requires yet another set of variables

$u=\sqrt{2 \Phi} \cos \phi, \quad U=-\sqrt{2 \Phi} \sin \phi$.

With $G=0$, the Hamiltonian $\mathcal{K}_{1}$ takes the form

$$
\begin{aligned}
\mathcal{K}_{1}= & -\frac{3}{8}\left(u^{2}+U^{2}\right)\left(4 A_{1}-4 \Psi C_{3}+\left(C_{2}+C_{3}\right)\left(u^{2}+U^{2}\right)\right) \\
& -\frac{C_{1}}{8}\left(2 \Psi-u^{2}-U^{2}\right)\left(U^{3}+3 u^{2}\right) .
\end{aligned}
$$

Equations of motion have a critical point $E_{4}$ at $u=U=0$ and the eigenvalues of variational equations are given by

$\lambda_{1,2}^{2}=-\frac{3}{4}\left(6 A_{1}+\Psi\left(C_{1}-6 C_{3}\right)\right)\left(2 A_{1}+\Psi\left(C_{1}-2 C_{3}\right)\right)$.

As it follows, the rectilinear polar orbits can be either stable or unstable. The orbits are unstable when

$\frac{2 A_{1}}{2 C_{3}-C_{1}}<\Psi<\frac{6 A_{1}}{6 C_{3}-C_{1}}$

and stable outside this interval. For prolate galaxies the lower limit becomes negative and all the orbits with

$\Psi<6 A_{1} /\left(6 C_{3}-C_{1}\right)$

are unstable. Referring to the previous results, we can observe that the lower limit in Eq. (70) is simply $\Psi_{23}$ from Eq. (57). From this point of view the stable equilibrium $E_{4}$ can be considered as the limit case of $E_{2}$.

\subsection{Summary}

Considering the mean variables $G$ and $\Psi$ as parameters, we can clearly distinguish up to four domains in the $(G, \Psi)$ plane, according to the existence and stability of various equilibria. Figure 2 presents the partition of the parametric plane for an oblate and a prolate galaxy, together with four exemplary phase space plots in the $(\phi, \Phi)$ Mercator chart. The latter are restricted to the $-\frac{\pi}{2} \leqslant \phi \leqslant \frac{\pi}{2}$ range, but thanks to symmetries, the remaining parts can be easily recovered by means of a simple horizontal translation. The areas below the $\Psi=|G|$ lines are meaningless (see Sect. 4).

Let us briefly review the qualitatively different types of the phase flow in the mean $(\phi, \Phi)$ variables. In the domain I of the parametric plane, only the equilibria $E_{0}$ and $E_{1}$ exist and both are stable. The equatorial $(\Phi=\Psi)$ orbits cannot be properly located in the Mercator chart, so we simply added a thick, continuous line above the upper edge to mark the existence and stability of $E_{0}$. A similar convention has been applied in other plots; if the line becomes dashed, the equilibrium $E_{0}$ is unstable.

One of the boundaries of the region I is the $\Psi=|G|$ line. The Fig. 2 phase flow plots top and bottom coincide in this case, i.e. we have equatorial, circular orbits. The equilibria $E_{1}$ and $E_{0}$ merge, resulting in a stable orbit, with one exception: a pair of $G= \pm \Psi_{3}$ points, where the joint equilibrium $E_{0}-E_{1}-E_{2}-E_{3}$ is unstable. This result agrees with the work of 

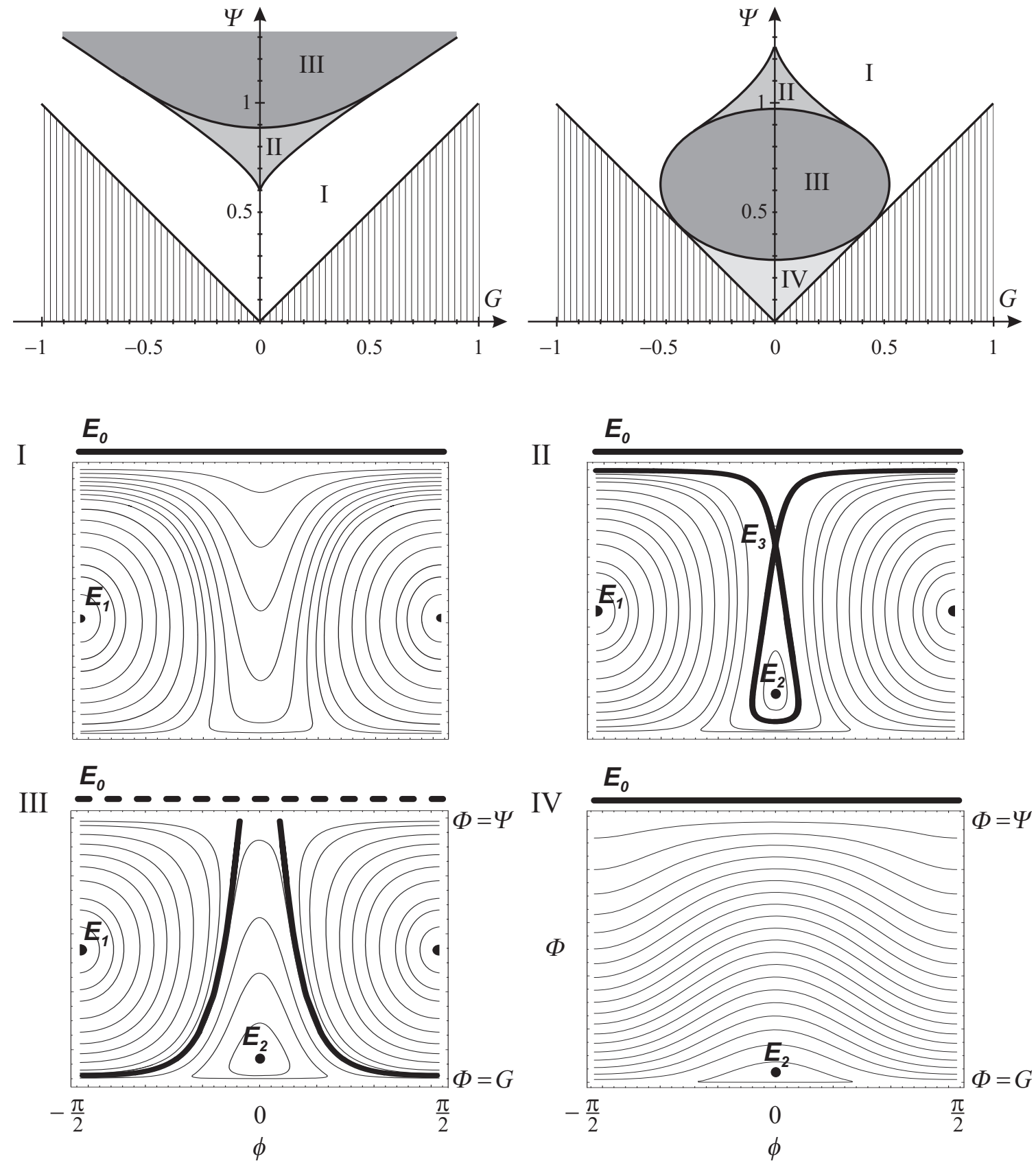

Fig. 2. Partition of the parametric plane for oblate (top-left, $e=0.4$ ) and prolate (top-right, $e=-0.4$ ) galaxies. Phase flow types in domains I-IV are shown below.

Binney (1981, Eq. (12a)), provided the present potential expressions are substituted and only his $a=1$ is considered. Crossing the tangent bifurcation line we move from region I to the region II, with its rich phase portrait containing $E_{0}, E_{1}$, $E_{2}$ and $E_{3}$ equilibria - only the last one being unstable.

The pitchfork bifurcation line surrounds the domain III, where only $E_{0}, E_{1}$ and $E_{2}$ exist. This is the only part of the parametric plane with unstable circular orbits.

The first three domains could be met for both the oblate and the prolate galaxies; the region IV, however, may exist only in the prolate case. In this domain we have two stable equilibria $E_{0}$ and $E_{2}$. Recalling the relation between $r, \Psi$, and $G$, we may conclude that many stars in oblate galaxies should have orbits of the type I, whereas in prolate galaxies the innermost orbits should belong to type IV, but further from the center types I and III become generic.

Figure 2 is incomplete in two aspects. It does not include inhomogeneous spherical galaxies (with $e=0$ ). But the dynamics in this case is quite simple: there are only two possible types of the phase flow depending on the value of $G$ being zero or not (see Fig. 3). The second point that requires a separate comment is the question of the $G=0$ axis that, strictly speaking, does not belong to any of the four domains in Fig. 2. However, one can simply obtain the phase flow picture at $G=0$ by shifting the $E_{2}$ equilibrium down to the bottom edge $\Phi=G=0$ of the plots I-IV in Fig. 2, where it turns into $E_{4}$. Like $E_{2}$, the $E_{4}$ equilibrium will remain stable in the regions II, III, and IV. In region $I$ the equilibrium $E_{2}$ does not exist and for $G=0$ the 


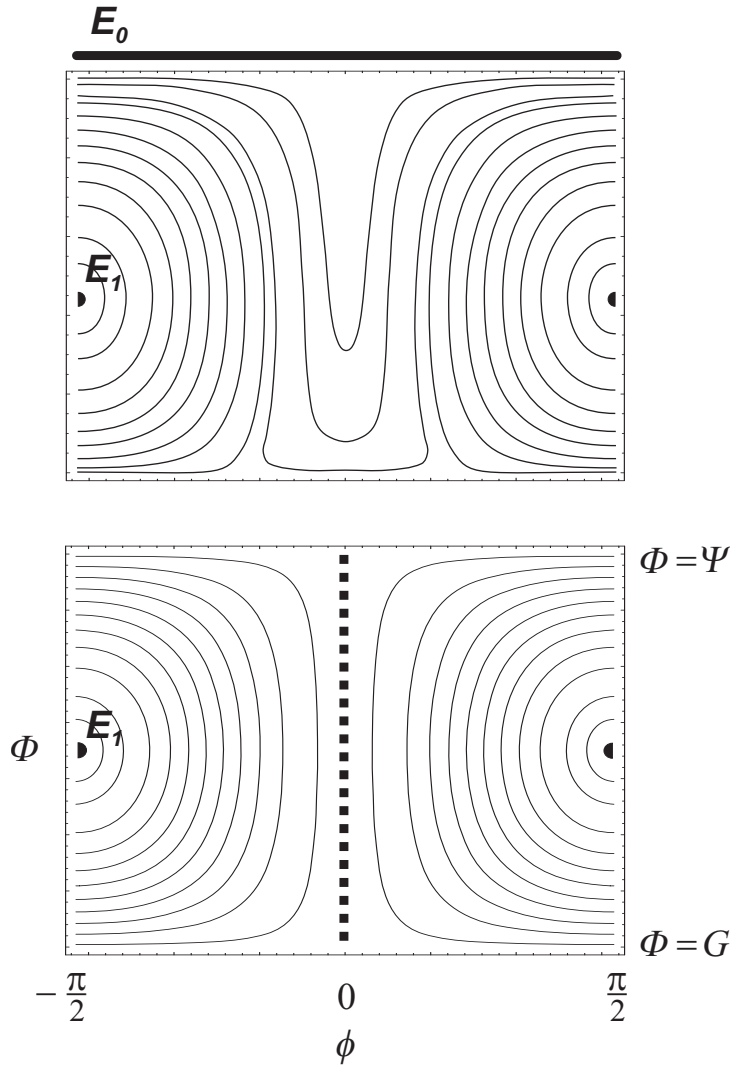

Fig. 3. Phase flow in an inhomogeneous spherical galaxy for $G \neq 0$ (top) and $G=0$ (bottom). The vertical dashed line consists of the neutral stability critical points.

polar rectilinear orbits $E_{4}$ are unstable, but not always! The exception to this rule is the region of small $\Psi$ for oblate galaxies, where $E_{4}$ becomes stable (see Fig. 4).

\section{Periodic orbits}

\subsection{Preliminaries}

Each equilibrium of the normalized Hamiltonian $\mathcal{K}$ is related to a family of periodic orbits inside a galaxy. But some families may exist only for particular values of the reference frame angular rate $\Omega$.

In order to discuss the periodic orbits within the framework of our first order solution, we need to know the short periodic perturbations in coordinates. They are obtained from the generator $\mathcal{W}$ defined by Eq. (36), using the definition (25) of rectangular variables and evaluating Poisson brackets with respect to the variables $\Xi^{*}$, so that

$x\left(\Xi^{*}\right)=x^{*}+\left\{x^{*}, \mathcal{W}\right\}$,

and similarly for the remaining variables. By $x^{*}$ we mean $x(\Xi)$ as defined in Eq. (25), but with all $\Xi$ actions and angles directly replaced by the mean variables $\Xi^{*}$. Thus we obtain

$$
\begin{aligned}
x= & \alpha_{1} \cos \left(\phi^{*}+\psi^{*}+g^{*}\right)-\alpha_{2} \cos \left(\phi^{*}+\psi^{*}-g^{*}\right) \\
& +\alpha_{3}\left[d^{*} \cos \left(\phi^{*}-\psi^{*}-g^{*}\right)-s^{*} \cos \left(\phi^{*}-\psi^{*}+g^{*}\right)\right] \\
& +\frac{\alpha_{3}}{2}\left[s^{*} \cos \left(\phi^{*}+3 \psi^{*}+g^{*}\right)-d^{*} \cos \left(\phi^{*}+3 \psi^{*}-g^{*}\right)\right] \\
& +\alpha_{4} s^{*} \cos \left(3 \phi^{*}+3 \psi^{*}+g^{*}\right) \\
& -\alpha_{4} d^{*} \cos \left(3 \phi^{*}+3 \psi^{*}-g^{*}\right) .
\end{aligned}
$$

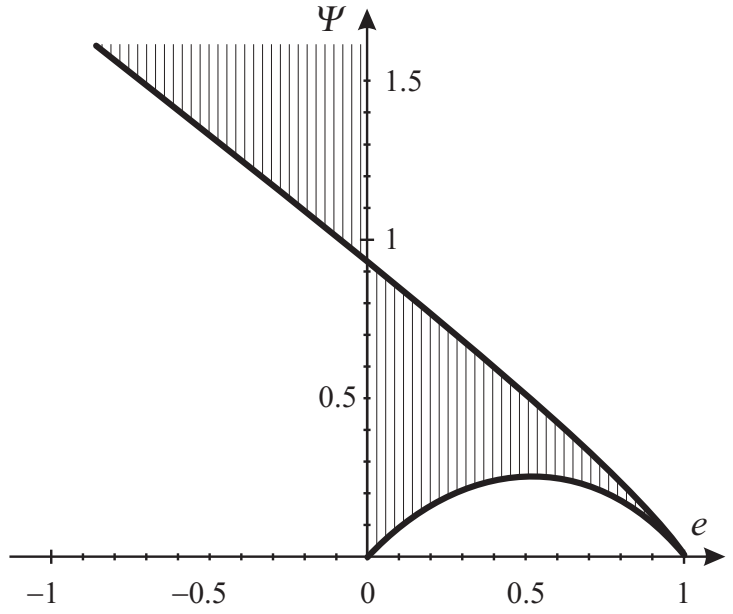

Fig. 4. Stability regions of rectilinear polar orbits $E_{4}$ implied by Eq. (70) as a function of galactic shape $e$ and mean $\Psi$. The orbits are unstable inside the shaded areas.

$$
\begin{aligned}
y= & \alpha_{1} \sin \left(\phi^{*}+\psi^{*}+g^{*}\right)+\alpha_{2} \sin \left(\phi^{*}+\psi^{*}-g^{*}\right) \\
& -\alpha_{3}\left[d^{*} \sin \left(\phi^{*}-\psi^{*}-g^{*}\right)+s^{*} \sin \left(\phi^{*}-\psi^{*}+g^{*}\right)\right] \\
& +\frac{\alpha_{3}}{2}\left[s^{*} \sin \left(\phi^{*}+3 \psi^{*}+g^{*}\right)+d^{*} \sin \left(\phi^{*}+3 \psi^{*}-g^{*}\right)\right] \\
& +\alpha_{4} s^{*} \sin \left(3 \phi^{*}+3 \psi^{*}+g^{*}\right) \\
& +\alpha_{4} d^{*} \sin \left(3 \phi^{*}+3 \psi^{*}-g^{*}\right) . \\
z= & \sqrt{\frac{d_{1}^{*}}{2}}\left[\left(2-2 A_{1}+3 d_{1}^{*} C_{3}+\Phi^{*} C_{1}\right) \sin \psi^{*}\right. \\
& +\frac{d_{1}^{*} C_{3}}{2} \sin 3 \psi^{*}+\frac{s^{*} d^{*} C_{1}}{2} \sin \left(2 \phi^{*}+3 \psi^{*}\right) \\
& \left.+s^{*} d^{*} C_{1} \sin \left(2 \phi^{*}+\psi^{*}\right)\right]
\end{aligned}
$$

where

$s^{*}=\sqrt{\frac{\Phi^{*}+G}{2}}, \quad d^{*}=\sqrt{\frac{\Phi^{*}-G}{2}}$,

and

$d_{1}^{*}=\Psi^{*}-\Phi^{*}$

$\alpha_{1}=\frac{s^{*}}{2}\left[2+A_{1}+d_{1}^{*} C_{1}+C_{2}\left(3 \Phi^{*}-G\right)\right]$,

$\alpha_{2}=\frac{d^{*}}{2}\left[2+A_{1}+d_{1}^{*} C_{1}+C_{2}\left(3 \Phi^{*}+G\right)\right]$,

$\alpha_{3}=\frac{C_{1} d_{1}^{*}}{4}$

$\alpha_{4}=\frac{C_{2} s^{*} d^{*}}{2}$.

We are also going to use the information about the angular rates

$\dot{\phi}^{*}=-3 A_{1}+C_{1}\left(\Phi^{*}-d_{1}^{*}\right)-3 C_{2} \Phi^{*}+3 C_{3} d_{1}^{*}$

$$
-C_{1}\left(\frac{d_{1}^{*} \Phi^{*}}{4 d^{*} s^{*}}-d^{*} s^{*}\right) \cos 2 \phi^{*}
$$

$\dot{g}^{*}=-\Omega+C_{2} G+\frac{C_{1} d_{1}^{*} G}{4 d^{*} s^{*}} \cos 2 \phi^{*}$,

$\dot{\psi}^{*}=1+2 A_{1}-C_{1} \Phi^{*}-3 d_{1}^{*} C_{3}-C_{1} d^{*} s^{*} \cos 2 \phi^{*}$.

We do not provide the expressions for the momenta $X, Y, Z$, but they follow a similar pattern as the coordinates $x, y, z$. 
All equilibria are related to the constant values of $\Phi^{*}, G$, and $\Psi^{*}$. Only $E_{1}, E_{2}$ and $E_{3}$ imply $\phi^{*}=$ const. in addition. The stability of each periodic orbit is the same as the stability of the associated equilibrium, thus it is not necessary to repeat the results of the previous sections.

\subsection{Periodic orbits independent of $\Omega$}

There are two kinds of periodic orbits that exist regardless of the reference frame rotation rate $\Omega$ : rectilinear polar orbits and circular equatorial orbits. Both kinds are invariant with respect to the $O z$ rotations.

The equilibrium $E_{4}$, that exists for $\Phi=G=0$, and hence $s^{*}=d^{*}=0$, defines a rectilinear polar orbit with $x=y=0$ and

$z=\sqrt{\frac{\Psi^{*}}{2}}\left[\left(2-2 A_{1}+3 \Psi^{*} C_{3}\right) \sin \psi^{*}+\frac{\Psi^{*} C_{3}}{2} \sin 3 \psi^{*}\right]$,

where

$\dot{\psi}^{*}=1+2 A_{1}-3 \Psi^{*} C_{3}=$ const.

The circular equatorial orbits can be seen as the special case of $E_{0}$, with $\Phi^{*}=\Psi^{*}=|G|$. In that case

$x= \pm \sqrt{\Psi^{*}}\left(1+\frac{A_{1}}{2}+C_{2} \Psi^{*}\right) \cos \left(\phi^{*}+\psi^{*} \pm g^{*}\right)$,

$y=\sqrt{\Psi^{*}}\left(1+\frac{A_{1}}{2}+C_{2} \Psi^{*}\right) \sin \left(\phi^{*}+\psi^{*} \pm g^{*}\right)$,

$z=0$,

and the frequency is

$\dot{\phi}^{*}+\dot{\psi}^{*} \pm \dot{g}^{*}=1 \mp \Omega-A_{1}-2 C_{2} \Psi^{*}$.

In the above expressions, the upper sign refers to the $G \geqslant 0$ case and the lower to $G<0$.

\subsection{Simple periodic orbits}

Inspecting the coordinates expressions (72)-(74) we notice that once we fix the value of $\phi^{*}$ at an equilibrium $E_{1}, E_{2}$, or $E_{3}$, the coordinates involve five distinct frequencies $v_{k}$ :

$v_{1}=\dot{\psi}^{*}+\dot{g}^{*}, \quad v_{2}=\dot{\psi}^{*}-\dot{g}^{*}, \quad v_{3}=3 \dot{\psi}^{*}+\dot{g}^{*}$, $v_{4}=3 \dot{\psi}^{*}-\dot{g}^{*}, \quad v_{5}=\dot{\psi}^{*}$.

The first four appear in $x$ and $y$, whereas $v_{5}$ and its multiples occur only in the expressions of the $z$ coordinate. Note that $v_{5} \neq 0$ according to the assumptions of the perturbation theory.

In general, any rational value of $\dot{g}^{*} / \dot{\psi}^{*}$ generates a periodic orbit because then all $v_{k}$ become rationally dependent on $v_{5}$ and the orbit becomes closed. The most important case, that we will call simple periodic orbits, arises when $\dot{g}^{*}=0$. The name is justified by the fact that orbits of this kind have a shape of symmetrically distorted ellipses (possibly degenerate), and a short period $T=2 \pi v_{5}^{-1} \approx 2 \pi$. We are going to consider separately the motion in the fixed $(\Omega=0)$ and in the rotating $(\Omega \neq 0)$ frames.

\subsubsection{Fixed frame $(\Omega=0)$}

Let us begin with an exceptional case of the equatorial orbits $E_{0}$, where we do not assume $\dot{\phi}^{*}=0$. At this equilibrium, $\Phi^{*}=\Psi^{*}$. Then, $d_{1}^{*}=0, z=0$, and coordinates $x, y$ depend only on the cosine or sine functions of $\left(\phi^{*}+\psi^{*}\right) \pm g^{*}$ and $3\left(\phi^{*}+\psi^{*}\right) \pm g^{*}$. By the assumptions of the perturbation theory,

$\dot{\phi}^{*}+\dot{\psi}^{*}=i^{*}=1-A_{1}-3 \Psi^{*} C_{2} \neq 0$.

This means that we have to impose $\dot{g}^{*}=0$ in order to obtain the periodic motion, but this is possible only if $G=0$. Thus the equatorial orbits are simple periodic in the fixed frame only if they degenerate into rectilinear equatorial orbits, or if they belong to the circular class discussed earlier. The rectilinear orbits are

$x=-\sqrt{\frac{\Psi^{*}}{2}} r_{1} \sin g^{*}$

$y=\sqrt{\frac{\Psi^{*}}{2}} r_{1} \cos g^{*}$,

$r_{1}=\left(2+A_{1}+3 C_{2} \Psi^{*}\right) \sin l^{*}+\frac{C_{2} \Psi^{*}}{2} \sin 3 l^{*}$,

and, obviously, $z=0$.

Periodic orbits at the $E_{1}$ equilibrium should have $\phi^{*}=\frac{\pi}{2}$ or $\phi^{*}=\frac{3 \pi}{2}$, and an appropriate value of $\Phi^{*}$. According to the definition $\phi^{*}=l^{*}-h^{*}$, this implies the following synchronicity: let $r_{\mathrm{e}}=\sqrt{x^{2}+y^{2}}$ stand for the equatorial projection of the radius vector; then the maximum of $r_{\mathrm{e}}$ is attained while the moving body crosses the equatorial plane and the minimum of $r_{\mathrm{e}}$ occurs when $|z|$ attains its maximum. For simple periodic orbits at $E_{1}$ we have to freeze the motion of the orbital plane seen in Fig. 1. The condition $\dot{g}^{*}=0$ reads

$G\left(C_{2}-\frac{C_{1} d_{1}^{*}}{4 d^{*} s^{*}}\right)=0$.

The case of $G=0$ refers to the polar orbits, whereas the vanishing term in the bracket defines an inclined orbit. In both cases the orbits are planar, oval shaped and have two perpendicular symmetry axes.

Simple periodic orbits associated with $E_{2}$ and $E_{3}$ are quite similar to the $E_{1}$ case. This time, however, the equatorial and polar motions are oppositely synchronized: $r_{\mathrm{e}}$ and $|z|$ attain their maxima and minima at the same time. The periodicity criterion differs from (87) only by one sign

$G\left(C_{2}+\frac{C_{1} d_{1}^{*}}{4 d^{*} s^{*}}\right)=0$,

but the consequences are significant. Noticing that all symbols in the bracket are non-negative, we conclude that only simple periodic orbits of the polar kind are generated by $E_{2}$ and $E_{3}$ in the fixed reference frame.

\subsubsection{Rotating frame $(\Omega \neq 0)$}

For the $E_{0}$ equatorial orbits, Eq. (85) remains true when $\Omega \neq 0$. As before, we can request $\dot{g}^{*}=0$, but now it means $G=\Omega / C_{2} \neq 0$. In the rotating reference frame, equatorial simple periodic orbits are no longer rectilinear, but they attain 
a symmetric, oval shape, approaching the circle when $|\Omega| / C_{2}$ tends to $\Psi^{*}$.

Similarly, the $E_{1}$ simple periodic orbits, described in the previous section, require $\dot{g}^{*}=0$ with

$C_{2}-\frac{C_{1} d_{1}^{*}}{4 d^{*} s^{*}}=\frac{\Omega}{G}$,

instead of Eq. (87). This means that periodic orbits of this type will not be polar.

As far as the periodic orbits related to $E_{2}$ and $E_{3}$ are concerned, the usual condition $\dot{g}^{*}=0$ becomes

$C_{2}+\frac{C_{1} d_{1}^{*}}{4 d^{*} s^{*}}=\frac{\Omega}{G}$

So, contrarily to the fixed frame case, the orbits are not polar. Also, that they exist only if $\Omega$ and $G$ have the same signs.

\subsection{Other periodic orbits}

There is a huge variety of periodic orbits in the discussed potential that are not simple. They attain the form of of closed planar or spatial rosettes (loops) with periods longer than $2 \pi$.

Equatorial orbits $E_{0}$ may become periodic when the ratio

$\frac{\dot{g}^{*}}{\dot{l}^{*}}=\frac{C_{2} G-\Omega}{1-A_{1}-3 \Psi^{*} C_{2}}=\frac{n_{1}}{n_{2}}$,

becomes a rational number with $n_{1}, n_{2} \in \mathbb{Z} \backslash\{0\}$. An $E_{1}$ orbit is periodic when the ratio

$\frac{\dot{g}^{*}}{\dot{\psi}^{*}}=\frac{G\left(C_{2}-\frac{C_{1} d_{1}^{*}}{4 d^{*} s^{*}}\right)-\Omega}{1+2 A_{1}+C_{1}\left(d^{*} s^{*}-\Phi^{*}\right)-3 C_{3} d_{1}^{*}}$,

is a rational number. Rational values of

$\frac{\dot{g}^{*}}{\dot{\psi}^{*}}=\frac{G\left(C_{2}+\frac{C_{1} d_{1}^{*}}{4 d^{*} s^{*}}\right)-\Omega}{1+2 A_{1}-C_{1}\left(d^{*} s^{*}+\Phi^{*}\right)-3 C_{3} d_{1}^{*}}$,

provide periodic $E_{2}$ and $E_{3}$ orbits.

The possibilities of obtaining a periodic orbit are so numerous that we will not investigate them in the present paper. One should note, however, that if the reference frame is fixed or rotates slowly, dense loops should be expected, because then $\dot{\psi}^{*}$ is much greater than $\left|\dot{g}^{*}\right|$.

\section{Conclusions}

The treatment we have presented is restricted to the periodic orbits generated by the 1:1 commensurability between the radial and longitudinal oscillations, although we have not used the epicyclic formulation explicitly. Thus, for example, we were not able to obtain the circular orbits with constant $z \neq 0$, nor the ones with different oscillation ratios. This limitation can be suppressed (or rather shifted) with a different choice of the canonical variables.

The normalization of the Hamiltonian was not complicated, but then we had to use four different variable sets in order to account for the geometric singularities associated with mapping the polar parametrization onto a cylindric surface. Nevertheless, three of the variable sets were only temporarily needed; it is a matter of further research to see if a proper family of manifolds exists that might allow the use of a single variable set in the averaged problem.

Depending on the values of $\Psi^{*}$ and $G \neq 0$ there are four qualitatively different phase portraits of motion. In other words, we have shown how the existence and stability of equilibria depends on the $z$ component of the angular momentum and on the mean unperturbed energy. The equilibria led us to the identification of periodic orbits in the vicinity of the 1:1 resonance. In the fixed reference frame, the following simple periodic orbits were found:

1. rectilinear polar orbits;

2. oval-shaped polar orbits;

3. oval-shaped tilted orbits (equatorially stretched);

4. circular equatorial orbits;

5. rectilinear equatorial orbits.

The longitudinal orientation of all the orbits is arbitrary, but it can be well expected that when the galaxy becomes slightly triaxial, only the two preferred directions will remain $(x$ and $y$ axes) as in the paper by de Zeeuw (1985).

When the reference frame rotates, the basic set of simple periodic orbits consists of

1. rectilinear polar orbits;

2. oval-shaped and circular equatorial orbits;

3. oval-shaped tilted orbits (equatorially stretched or shrunken).

We have pointed out a possibility of other types of orbits related to the commensurability (or simply "the resonance") between - roughly speaking - the rotation of the reference frame and radial/vertical oscillations. But if we attach the physical meaning to the reference frame and request that it rotates with the galaxy, the rotation rates $\Omega$ are slow (Davoust 1986; Martinet $\&$ de Zeeuw 1988). Accordingly, these secondary resonances will be of quite a high order and of marginal significance for the dynamics.

Acknowledgements. This paper has been partially supported by the Spanish Ministry of Science and Technology (Project ESP2002-02329) and by the Polish State Committee for Scientific Research (KBN 5T12D02623 grant). The authors appreciate the comments of the anonymous reviewer.

\section{References}

Binney, J. 1981, MNRAS, 196, 455

Binney, J., \& Tremaine, S. 1987, Galactic Dynamics (Princeton Univ. Press)

Contopoulos, G. 1960, Z. Astrophys., 49, 273

Contopoulos, G., \& Moutsoulas, M. 1965, AJ, 70, 817

Davoust, E. 1983, A\&A, 125, 101

Davoust, E. 1986, A\&A, 156, 152

de Zeeuw, T. 1985, MNRAS, 215, 731

Deprit, A. 1969, Celest. Mech., 1, 12

Deprit, A. 1991, Celest. Mech. and Dynamical Astron., 51, 201

Ferrers, N. M. 1877, Quart. J. Pure Appl. Math., 14, 1

Gustavson, F. G. 1966, AJ, 71, 670

Jalali, M. A., \& Sobouti, Y. 1998, Celest. Mech. and Dynamical Astron., 70, 255

Martinet, L., \& de Zeeuw, T. 1988, A\&A, 206, 269

Ollongren, A. 1962, Bull. Astron. Inst. Netherlands, 16, 241

Whittaker, E. T. 1944, A Treatise on the Analytical Dynamics of Particles and Rigid Bodies (New York: Dover) 